\title{
Mathematical model of vibrating air pump unit
}

\author{
Fakhriddin Bekchanov ${ }^{*}$, Rustam Ergashev, Tulkin Mavlanov and Oleg Glovatskiy \\ Tashkent Institute of Irrigation and Agricultural Mechanization Engineers, 100000, Kari Niyaziy
}

\begin{abstract}
Based on the laws of classical mechanics, in particular, the law of conservation of momentum, the paper describes the developed mathematical model of signal propagation during vibration diagnostics. At the beginning, the problem of signal propagation was investigated, which was reduced to solving the problem of wave propagation. According to the analysis of experimental results investigated, that the attenuated nature of the signals must be taken into account. For this purpose, a mathematical model has been developed, which allows to solve the problem of the propagation of damped signals. Comparative analysis allows to conclude that the constructed model is adequate.
\end{abstract}

\section{Introduction}

Currently in the Republic of Uzbekistan about $70 \%$ of the land is irrigated using centrifugal pumps, the smooth operation of these pumps is necessary for the successful development of agriculture. In the Action Strategy for the further development of the Republic of Uzbekistan for 2017-2021, special attention is paid to the development of land reclamation and irrigation facilities to increase the level of the national economy". The implementation of this task, aimed at improving the accuracy of diagnosing the state of pumping units, becomes important.

\section{Methods}

As a rule, when vibrodiagnostics using an accelerometer installed at certain points of the machinery, a signal is recorded in the form of vibration acceleration. This signal, either in the device, which is called the vibrator, or integrated in the computer, is converted into vibration velocity or vibration displacement (Fig. 1). All these three types of signals in vibration diagnostics are considered periodic polyharmonic processes.

The leading direction in vibration diagnostics is the analysis of the spectrum of the vibration signal.

When constructing a mathematical model, we will proceed from the laws of theoretical mechanics, in particular, from the law of conservation of momentum, which leads to the equation of signal propagation in the form of vibration accelerations in integral form [1]. In order to pass from an integral equation to a differential one, suppose that the desired function has second derivatives.

The task of signal propagation is reduced to solving a differential equation of the form:

\footnotetext{
* Corresponding author: faxriddinbekchanov@ mail.ru
} 


$$
\frac{\partial^{2} u}{\partial t^{2}}=a^{2} \frac{\partial^{2} u}{\partial x^{2}}
$$

satisfying homogeneous boundary conditions at:

$$
u(o, t)=0 ; u(l, t)=0
$$

And initial conditions:

$$
u(x, o)=\varphi(x) ; \dot{u}(x, o)=\psi(x)
$$

To solve the problem using the method of separation of variables. According to this method, equation (1) is represented as:

$$
u(x, t)=X(x) Y(t)
$$

where: $\mathrm{X}(\mathrm{x})$ is a function of variable $\mathrm{x}$ only;

$$
\mathrm{Y}(\mathrm{t}) \text { - function of variable t only. }
$$

As a result of substituting equation (4) into equation (1), we obtain the following expression:

$$
\frac{X^{\prime \prime}(x)}{X(x)}=\frac{1}{a^{2}} \frac{\ddot{Y}(t)}{Y(t)}=-\lambda ;
$$

where: $\lambda$ is a constant, which, for the convenience of subsequent calculations, is taken with a minus sign, without assuming anything about its sign.

From the expression (5) we obtain the differential equations for determining.

$$
\begin{array}{r}
X(x) \text { and } Y(t) ; \\
X^{\prime \prime}(x)=\lambda X(x)=0 \\
\ddot{Y}(t)=a^{2} \lambda Y(t)=0
\end{array}
$$

Moreover, the boundary and initial conditions are taken as zero.

It is determined [2] that when the value of $\lambda 1$ is equal to

$$
\lambda_{n}=\left(\frac{\pi \cdot n}{l}\right)^{2}
$$

There are nontrivial solutions of the problem (1)

$$
X_{n}(x)=\sin \frac{\pi \cdot n}{l} x
$$

Defined up to an arbitrary factor, which we set equal to one. The same values of $\lambda_{n}$ correspond to the solution of equation (7) in the form.

$$
y(t)=\frac{a_{o}}{2}+\sum_{k=1}^{\infty}\left(a_{k} \cos \left(\frac{2 \pi k}{T} t\right)+b_{k} \sin \left(\frac{2 \pi k}{T} t\right)\right),
$$

where: flourier coefficient for the k-th harmonic 


$$
\begin{gathered}
a_{k}=\frac{2}{T} \int_{O}^{T} y(t) \cos \left(\frac{2 \pi k}{T} t\right) d t, \quad b_{k}=\frac{2}{T} \int_{O}^{T} y(t) \sin \left(\frac{2 \pi k}{T} t\right) d t \\
y(t)=A_{O}+\sum_{k=1}^{\infty} A_{k} \cos \left(\frac{2 \pi k t}{T}-\varphi_{k}\right), A_{O}=\frac{a_{o}}{2}
\end{gathered}
$$

- average value.

Equations (10) can also be represented as

$$
Y(t)=A_{o}+\sum_{k=1}^{\infty} A_{k} \cos \left(\frac{2 \pi k t}{T}-\varphi_{k}\right), A_{o}=\frac{a_{o}}{2} ;
$$

where: $A_{k}=\sqrt{a_{k}^{2}+b_{k}^{2}}$ - module of the k-th harmonic of the spectrum;

$$
\varphi_{k}=\operatorname{arctg}\left(\frac{b_{k}}{a_{k}}\right)
$$

When $\mathrm{k}=1$ we have $f_{1}=\frac{1}{T}(\mathrm{~Hz})$ or circular frequency

$$
\omega_{1}=\frac{2 \pi}{T}
$$

Further, by virtue of linearity and homogeneity, we represent equation (1) as the sum of particular solutions:

$$
u(x, t)=\sum_{n=1}^{\infty}\left(A_{n} \cos \frac{\pi \cdot n}{l} a \cdot t+B_{n} \sin \frac{\pi \cdot n}{l} a \cdot t\right) \sin \frac{\pi \cdot n}{l} x
$$

This solution satisfies this equation and the boundary conditions.

However, due to the decaying nature of signal propagation, in general, the signal propagation equation can be represented as

$$
A_{o} \frac{\partial^{u} y}{\partial x^{u}}+A \frac{\partial^{2} y}{\partial x^{2}}+2 B \frac{\partial^{2} y}{\partial x \partial t}+C \frac{\partial^{2} y}{\partial t^{2}}+D \frac{\partial y}{\partial x}+E y=0
$$

where: $A_{o}=-\frac{E_{o} y}{T} ; \quad A=V_{1}^{2}+V_{0}^{2} ; \quad B=-V_{0} ; \quad C=-1 ;$

$$
D=\frac{\partial v_{0}}{\partial t}+\left(\frac{\partial P}{\partial x}\right) T \quad E=\omega_{e}^{2} ; \quad V_{n}^{2}=\frac{P}{T}
$$

In particular, from equation (12) one can get equation (1), however, due to the fading nature of signal propagation, the third term of equation (12) cannot be neglected.

In this case, the problem is reduced to solving an ordinary differential equation:

$$
\frac{d^{2} y_{n}}{d \cdot t^{2}}+B_{n} \frac{d \cdot y_{n}}{d \cdot t}+C_{n} y_{n}=F_{n}(t)
$$

To find a unique solution of the differential equation (14) in partial derivatives, it is necessary to determine the initial and boundary conditions.

Initial and boundary conditions are called the conditions specified at the initial time t. The boundary conditions are specified for different values of spatial variables.

Equation (14) is solved numerically under initial conditions. 


$$
t=0 ; \quad y_{n}=y_{n}(0) ; \quad \dot{y}_{n}=\dot{y}_{n}(0)
$$

To test the adequacy, we compare the results of solving equation (14) with the data of experimental studies and present them in the form of an accelerogram (Fig. 1.)

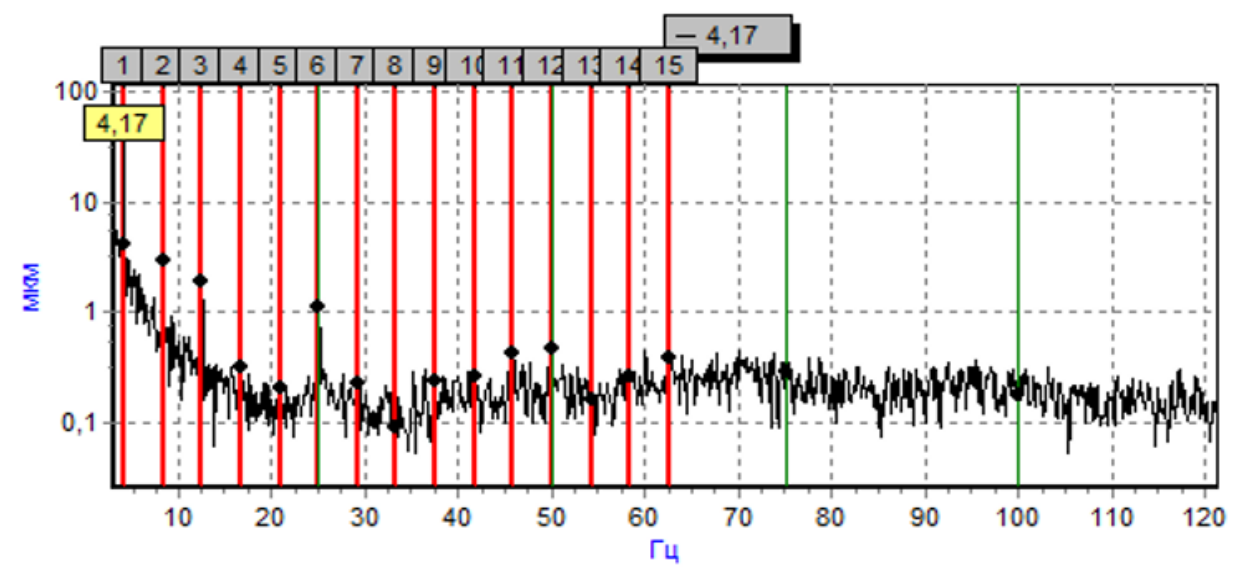

Fig. 1. Accelogram amplitude spectrum of vibration on the camera impeller pumps first Karshi Machine channel.

Using the "Spline interpolation" method, we replace the accelelogram with broken curves (Fig. 2).

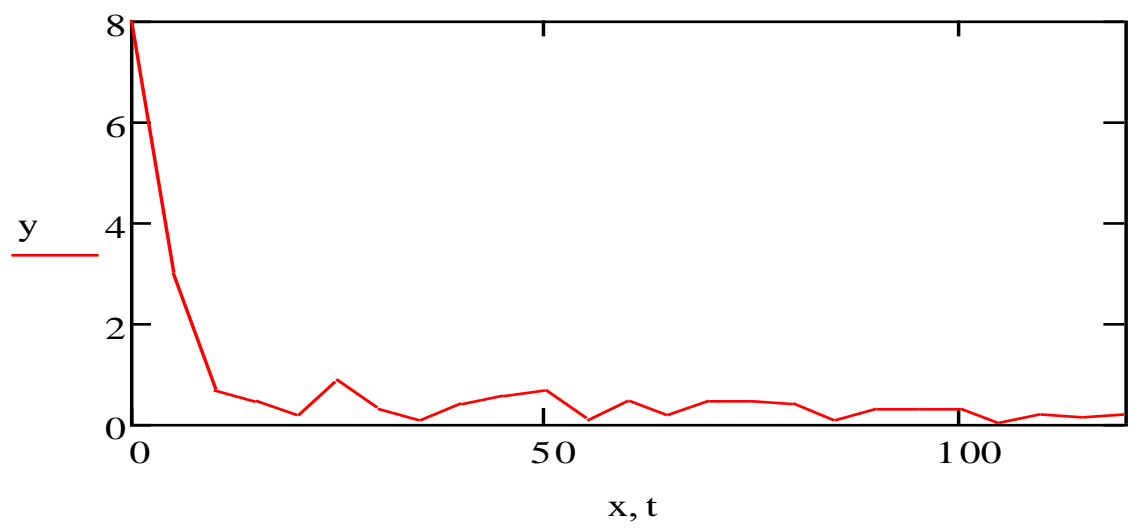

Fig. 2. Experimental result.

Further, according to the well-known method [2], we average the values and as a result we obtain the averaged curve (Fig. 3). 


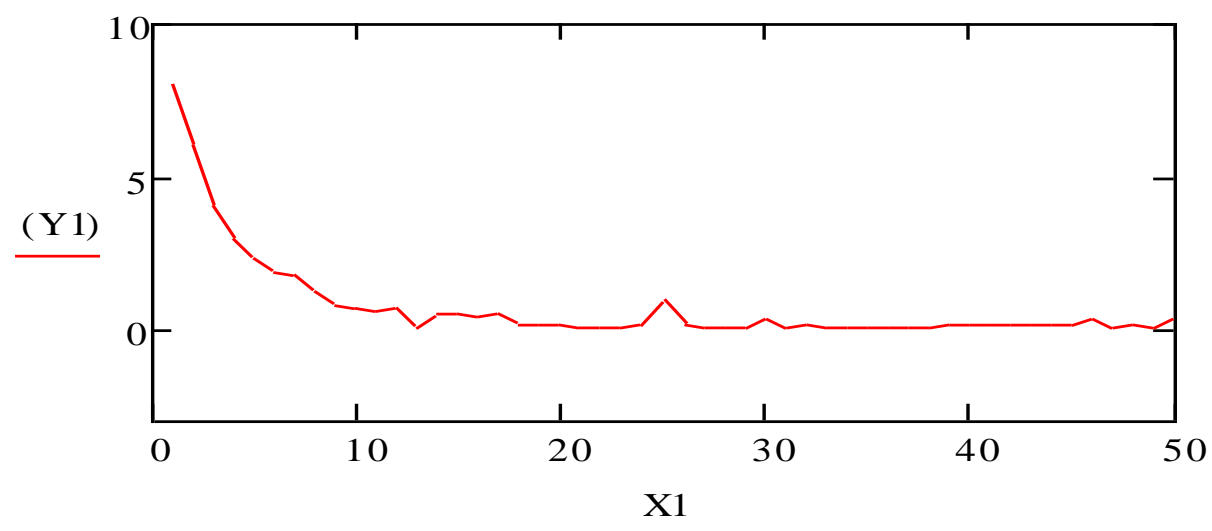

Fig. 3. Approximation of experimental results.

Based on the analysis and processing of experimental data with a given degree of accuracy, it is possible to assert that equation (14) is valid.

In case of replacement by a differential equation of the first order.

$$
\frac{d \cdot z}{d \cdot t}=z(t)+\sin (z(t), t) \quad x(0)=8
$$

The pattern of signal change is shown in Fig. 4.

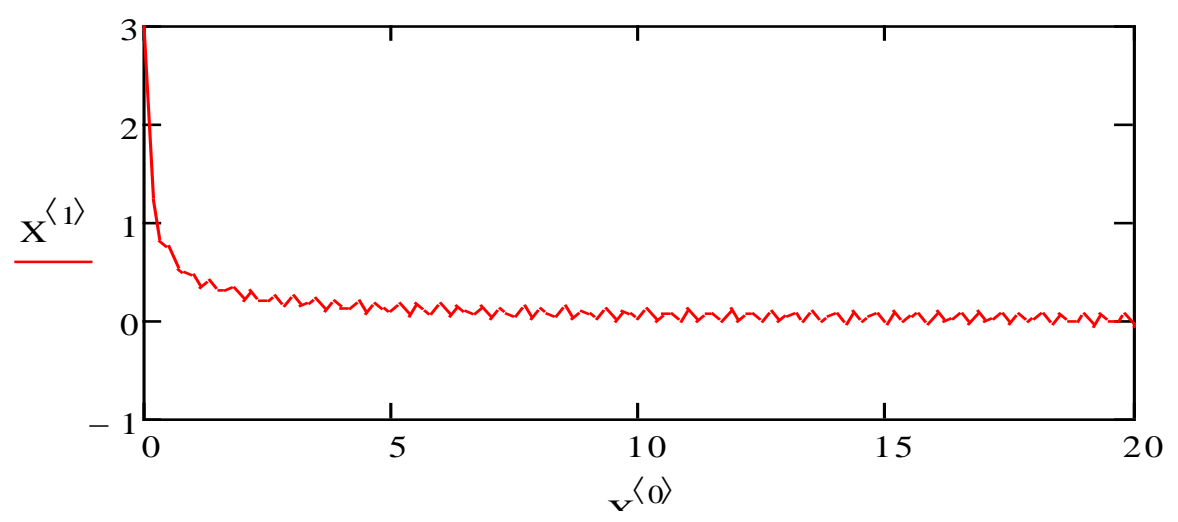

Fig. 4. The pattern of changing signal.

Comparing the experimental and theoretical curves, we can conclude about the adequacy of the mathematical model.

Next, go to the complex values.

For a periodic function of a Fourier series in a complex form, has the form:

$$
\begin{aligned}
& y(t)=\sum_{k=-\infty}^{\infty} c_{k} \exp \left[\left(\frac{i 2 \pi k}{T}\right) t\right] \\
& \text { where } \\
& c_{r}=\frac{\int_{O}^{T} y(t) \exp \left[\left(-\frac{i 2 \pi k}{T}\right) t\right] d t}{T},
\end{aligned}
$$




$$
c_{k}=\frac{\left(a_{k}-i b_{k}\right)}{2} ; \quad c_{-k}=\frac{\left(a_{k}+i b_{k}\right)}{2} ;
$$

(coefficients $\mathrm{c}_{\mathrm{k}}$ complex conjugate). Relationship (15) is based on the Euler formula

$$
e^{-i x}=\cos x-i \sin x
$$

An important characteristic of the vibration signal is the root mean square value (RMS).

Given the parseval equality, equations (15) take the following form:

$$
\frac{\int_{O}^{T} y^{2}(t) d t}{T}-\left[\frac{\int_{O}^{T} y^{2}(t) d t}{T}\right]^{2}=\sum_{k=1}^{\infty} \frac{a_{k}^{2}+b_{k}^{2}}{2}
$$

score of this great

$$
R M S=\sqrt{\frac{\sum_{k=1}^{L} A^{2}(k)}{2}} ;
$$

where: $\mathrm{L}$ is the number of lines of the spectrum.

Let the function y (t) be given at times $t_{i}=y\left(t_{i}\right)$, where: $t_{i}=i \cdot \Delta t ; \Delta t$ - sample rate, $\Delta t=\frac{T}{N} ; i=1, \ldots N, N$ - number of ordinates in function; $T$ - implementation length of the studied function. Further (ti) we will denote as y (i) or ui, i.e. i- e function value

The calculation of the spectrum module is performed by the formula:

$$
A_{k}=\left(Y\left(\omega_{k}\right)=\sqrt{a_{k}^{2}+b_{k}^{2}} ;\right.
$$

where is the frequency of the k-th harmonic $\omega_{k}=k \cdot \omega_{1}, f_{k}=\frac{k}{T}$; the coefficients $a_{k}$ and $b_{k}$ are not calculated by the formula, but by a numerical method. So, for example, by the method of rectangles

$$
a_{k}=\frac{2}{N} \sum_{i=1}^{N} y(i) \cos \left(\frac{2 \pi}{N} k i\right), b_{k}=\frac{2}{N} \sum_{i=1}^{N} y(i) \sin \left(\frac{2 \pi}{N} k i\right), \frac{a_{o}}{2}=\frac{\sum_{i=1}^{N} y(i)}{N},
$$

Function at sampling points

$$
y(i)=\frac{a_{o}}{2}+\sum_{k=1}^{N / 2} a_{k} \cos \left(\frac{2 \pi}{N} k i\right)+\sum_{k=1}^{N / 2-1} b_{k} \sin \left(\frac{2 \pi}{N} k i\right) .
$$

In complex form, this value is

$$
\begin{aligned}
& y(i)=\sum_{k=-(N / 2-1)}^{N / 2} c_{k} \exp (i 2 \pi k i / N), \\
& \text { where } \quad c_{k}=\frac{\sum_{i=1}^{N} y(i) \exp (-i 2 \pi k i / N) .}{N}
\end{aligned}
$$

Considering that $\mathrm{c}_{\mathrm{k}}=\mathrm{c}_{\mathrm{k}}$ 


$$
y(i)=\sum_{k=0}^{N-1} c_{k} \exp (i 2 \pi k i / N)
$$

In the future, the amplitude of the k-th harmonic, calculated by the formula (6), we will denote $\mathrm{Y}(\mathrm{k})$.

If the signal is not periodic, then its spectrum is continuous and is determined by the direct Fourier transform:

$$
s(\omega)=\int_{O}^{T} y(t) \exp (-j \omega t) d t
$$

The values of the function are determined by the inverse Fourier transform.

At discrete frequencies $f_{k}=\frac{k}{t} ; \quad s\left(\frac{k}{t}\right)=T \cdot c_{k} ;$

where: $c_{k}$ is the complex Fourier coefficients (the complex coefficient is equal to the area of the rectangle with base $f_{1}$ and height $s(k / T)$ ).

Based on the Kotelnikov theorem, if the signal has a frequency-limited spectrum $\left(f \leq F_{b}\right)$, then to restore the signal it is enough to know the spectrum at discrete points $f_{k}$.

Given the discreteness of the representation of the original signal y (i) the desire to represent the spectral density in discrete form, use the discrete Fourier transform (DFT) [3] (with the assumption that the values of $\mathrm{y}_{\mathrm{i}}$ )

$$
s_{k}=s(k / T)=\sum_{i=1}^{N} y(i) \exp (i 2 \pi k i / N), k=0,1, \ldots N-1
$$

At

$$
\begin{gathered}
N / 2 \leq k \geq(N-1) s_{k}=S_{N-k} \\
y(i)=\sum_{k=o}^{N-1} s(k) \exp (i 2 \pi k i / N) / N, i=1, \ldots, N \\
c_{k}=c(k / T)=(1 / T) \sum_{i=1}^{N} y(i) \exp (-i 2 \pi k i / N), k=0,1, \ldots N-1
\end{gathered}
$$

If the cutoff frequency $F c=N /(2 \Delta t)$ is significant, then the number of ordinates $\mathrm{N}$ at a constant discretization step must also be large, which increases the amount of DFT calculations (the number of calculations is proportional to N2).

In this regard, in practice the methods of fast Fourier transform (FFT) are widely used (4)

\section{For FFT}

$$
X(k)=N \cdot C(k) ; \quad A(k)=Y(k)=(2 / N) \cdot X(k)
$$

We introduce such notation:

$\mathrm{f}_{\mathrm{g}}$ - sampling frequency $(\mathrm{Hz})$, sample rate $\Delta t=1 / \mathrm{fg}$ (sec), sale length $\mathrm{T}=\mathrm{N} / \mathrm{fg}$; sec

$\mathrm{f}_{1}$ - first harmonic frequency $(\mathrm{Hz}), \mathrm{f}_{1}=\frac{1}{\mathrm{~T}}=\frac{\mathrm{f}_{\mathrm{g}}}{\mathrm{N}} ; \mathrm{f}_{\mathrm{k}}=\mathrm{k} ; \mathrm{f}_{1}$ - frequency of the $\mathrm{k}$-th

harmonic (the realization length $\mathrm{T}$ determines the accuracy of the spectrum reproduction);

$\mathrm{L}$ - the number of lines in the spectrum 


$$
L=\frac{F_{b}}{f_{1}}=\frac{F_{b} \cdot N}{f_{g}}
$$

where: $F_{b}-$ high frequency. Based on the Kotelnikov theorem, $f_{g}>2 F_{b}$, often take $f_{g}=2,56$ $\mathrm{F}_{\mathrm{b}}\left(256=2^{8}\right)$;

$\mathrm{F}_{\mathrm{H}^{-}}$lower frequency $(\mathrm{Hz})$ if $\mathrm{T}>1 / \mathrm{F}_{\mathrm{H}}$ that $\mathrm{f}_{1}<\mathrm{F}_{\mathrm{H}}$;

$\mathrm{f}_{\mathrm{p}}$ - rotor speed $(\mathrm{Hz})$;

\section{Discussion}

Consider a practical case of importance:

$\mathrm{f}_{\mathrm{g}}=2,56 \cdot \mathrm{F}_{\mathrm{b}} \mathrm{Hz}, \mathrm{N}=2^{\mathrm{p}}$. Then $\mathrm{K}=1002^{\mathrm{p}-8}$ : at $\mathrm{p}=10(\mathrm{~N}=1024) \mathrm{L}=400$, at $\mathrm{p}=11 \quad(\mathrm{~N}=2048)$ $\mathrm{L}=800$, at $\mathrm{p}=12(\mathrm{~N}=4096) \mathrm{L}=1600$, at $\mathrm{p}=13(\mathrm{~N}=8192) \mathrm{L}=3200$. As we see, if $\mathrm{f}_{\mathrm{g}}>2 \cdot \mathrm{F}_{\mathrm{b}}$, then the number of lines of the spectrum $\mathrm{L}<\mathrm{N} / 2$.

If additionally $\mathrm{F}_{\mathrm{b}}=5000 \mathrm{~Hz}\left(\mathrm{f}_{\mathrm{g}}=12800 \mathrm{~Hz}\right)$, that $\mathrm{T}=2^{\mathrm{p}-7} / 100 ; \mathrm{f}_{1}=100 / 2^{\mathrm{p}-7} ; \mathrm{f}_{\mathrm{k}}=\mathrm{k} \cdot 100 / 2^{\mathrm{p}-7} ;$ relative harmonic number in rotor frequency $\mathrm{m}_{\mathrm{k}}=\mathrm{f}_{\mathrm{k}} / \mathrm{f}_{\mathrm{p}}=\mathrm{k} / 2^{\mathrm{p}-8}\left(\mathrm{f}_{\mathrm{p}}=50 \mathrm{~Hz}\right)$. At $\mathrm{p}=11 \mathrm{~m}_{1}=1 / 8$, $\mathrm{m}_{2}=1 / 4, \mathrm{~m}_{3}=3 / 8, \mathrm{~m}_{4}=1 / 2, \mathrm{~m}_{5}=5 / 8, \mathrm{~m}_{6}=3 / 4, \mathrm{~m}_{7}=7 / 8, \mathrm{~m}_{8}=1, \mathrm{~m}_{9}=9 / 8, \ldots, \mathrm{m}_{800}=100 ; \mathrm{m}_{\mathrm{k}}=1$ at $\mathrm{f}_{\mathrm{k}}=\mathrm{f}_{\mathrm{p}}$. If a $\mathrm{f}_{\mathrm{p}}=50 \mathrm{~Hz}$, that $\mathrm{k}=2^{\mathrm{p}-8}$, at $\mathrm{p}=11 \mathrm{k}=8$.

Comment: number of lines in increments $\mathrm{f}_{\mathrm{p}} \mathrm{L}_{\mathrm{p}} / 2 \mathrm{p}^{-8}=100$ and does not depend on $\mathrm{p}$. For sampling rate $12,8 \mathrm{KHz}$, the frequency step for the spectrum is: at $\mathrm{N}=1024 \mathrm{f}_{1}=12,5 \mathrm{~Hz}$, at $\mathrm{N}=2048 \mathrm{f}_{1}=6,25$, at $\mathrm{N}=4096 \mathrm{f}_{1}=3,125 \mathrm{~Hz}$, at $\mathrm{N}=8192 \mathrm{f}_{1}=1,5625 \mathrm{~Hz}$.

RMS value is determined as follows [4]:

$$
R M S=\left[\int_{0}^{T} y^{2}(t) d t / T\right]^{\frac{1}{2}}=\left[\sum_{i} y_{i}^{2} / N\right]^{\frac{1}{2}} ;
$$

Actually, formula (28) is valid in the case, if the constant component a $0 / 2$ equals zero. In vibration diagnostics vibration, removed from machinery, Filtered at low and high frequencies and set in the range ( $\mathrm{FH}, \mathrm{Fb}) \mathrm{FH}>0$, so the formula (28) is true for the time function (wave) and coincides in meaning with the formula (19), fair to the spectrum.

In the case when the vibration signal breaks up in time into parts, either when integrating or defining an RMS envelope, you must first remove the constant component

$$
R M S=\left[\sum y_{i}^{2} / N-\left(\sum y_{i} / N\right)^{2}\right]^{\frac{1}{2}} ;
$$

The number of terms in the formula (29) is equal to $\mathrm{L}$.

In addition to the VHC, in vibration diagnostics is used:

a) peak value - the largest absolute value of the maximum deviations of the oscillating quantity. There is a positive peak value and a negative peak value;

b) span - the difference between the highest and lowest values of the oscillating value.

RMS is the most important indicator, since it takes into account the temporal development of the studied fluctuations, and it directly displays the value associated with the signal energy and, therefore, the destructive power of these oscillations.

\section{Conclusions}

Comparison of the obtained results with experimental data allows us to conclude that, that the proposed method adequately determines the process under consideration, signal propagation. 


\section{References}

1. A.N. Tikhonov, A.A.Samara, Equations of mathematical physics. (Moscow: Science) pp. 736, (1977)

2. T.M. Mavlanov, N.V. Dremova, G.B. Abdieva. The dynamic strength of the elements of textile machines on the effect of a real oscillogram. pp. 28, (2003)

3. J. Bendat, A. Pirsol. 1983 Applications of correlation and spectral analysis. - Trans. from English., (Moscow: Mir) pp. 312 (1987)

4. V.G Kartashov, Fundamentals of the theory of discrete signals and digital filters. (Moscow: Higher School), (1982)

5. O.Y.Glovatsky, R.R. Ergashev, F.A. Bekchanov Analysis of diagnostics of pumping units of Jizzakh head pumping station. (Tashkent: Irrigation and melioration 3 (9), pp 32-34, (2017)

6. O.Y. Glovatsky, R.R. Ergashev, F.A. Bekchanov. Results of diagnostics of pump units. (Tashkent: Journal of Irrigation and melioration 1 (11), pp. 36-39, (2018)

7. F.A. Bekchanov, New methods for diagnosing pumps hydrotechnical systems. Delhi: International journal for innovative research in multidisciplinary fields. Vol. 4, Issue10), pp. 367-373, (2018)

8. O.Y. Glovatsky, R.R. Ergashev, F.A. Bekchanov, Sh.R. Rustamov, Estimation of the technical condition and availability of pumping units. (Almaty: International Agroengineering, Scientific and Technical Journal) pp. 64-67, (2012)

9. F.A. Bekchanov, Vibration measuring equipment in pumps. (Tashkent: Increase efficiency, reliability and safety of hydraulic structures. Republican Scientific and Practical Conference. TIIM) pp. 30-32, (2012)

10. F.A. Bekchanov, Diagnostic device for pumps units. (Andijan: International scientificpractical conference on "Problems of increasing the efficiency of production and energy efficiency of modern production.) pp. 1071-1074, (2018)

11. F.A. Bekchanov, Vibro diagnostic system software (Tashkent: International scientificpractical conference. Problems of increase of energy efficiency in agrarian sector.) pp. 212-219, (2018)

12. F.A. Bekchanov, Providing safe operation of pump units by diagnostic method (Tashkent: International scientific-practical conference "Problems of increase of energy efficiency in agrarian sector) pp. 286-291, (2018)

13. R.R. Ergashev, O.Y. Glovatsky, F.A. Bekchanov, N.R. Nasyrova, New methods of diagnosing large vertical pumping units "Improvement of reclamation status of irrigated lands and problems of efficient use of water resources", T., pp. 397-402, (2015)

14. T.Sh Majidov, R.R. Ergashev, F.A. Bekchanov, J.I. Rashidov, Improvement of the system of diagnostics of pump assemblies, International scientific-practical conference "Problems of increasing the efficiency of using electric energy in the branches of the agro-industrial complex", pp. 278-281, (Tashkent, 2015)

15. T.Sh. Majidov, R.R. Ergashev, F.A. Bekchanov, Determination the amount of vibration in pump unit. Republican scientific-practical conference "Ecological problems of rational use of water and land resources in irrigated farming". (2017)

16. R.R. Ergashev, F.A. Bekchanov, Pump units reliable operation, International scientificpractical conference on "Efficiency, reliability and safety of hydraulic engineering constructions", pp. 212-215, (2018) 